The yolk-shell structure bi-functional catalyst catalyzes the glucose to produce ethylene glycol

\author{
Qianghua Xin ${ }^{\text {a }}$, Shitao Yu ${ }^{\text {a }}$, Long Jiang ${ }^{\text {b }}$, Defu Yin ${ }^{\text {a }}$, Lu Li a , Congxia Xie ${ }^{\text {a }}$, \\ Qiong $\mathrm{Wu}^{\text {a }}$, Hailong $\mathrm{Yu}^{\text {a }}$, Yuxiang Liu ${ }^{\mathrm{a}}$, Yue Liu ${ }^{\text {a*, c }}$, Shiwei Liu ${ }^{\text {a*}} \square$ \\ a State Key Laboratory Base of Eco-chemical Engineering, College of Chemical Engineering, Qingdao \\ University of Science and Technology, 53 Zhengzhou Road, Qingdao 266042, Peoples Republic of
}

China

${ }^{b}$ CAS Key Laboratory of Bio-based Materials, Qingdao Institute of Bioenergy and Bioprocess

Technology, Chinese Academy of Sciences, Qingdao 266101, China

c Jiangsu Key Lab Biomass Energy \& Mat, Nanjing 210042, Jiangsu, Peoples R China

\title{
S1. Preparation of $\mathrm{MSiO}_{2}$ hollow nanospheres
}

The $30 \mathrm{~mL}$ water, $10 \mathrm{~mL}$ ethanol, $0.16 \mathrm{~g} \mathrm{CTAB}$ and $0.5 \mathrm{~mL}$ ammonia were added to $150 \mathrm{~mL}$ three-necked flask. The mixed solution was kept at $50^{\circ} \mathrm{C}$ for 30 minutes, then added $0.16 \mathrm{~mL}$ TEOS. After stirring for 30 minutes, TEOS $(0.24 \mathrm{~mL})$ was added again. Then the temperature was raised to $80^{\circ} \mathrm{C}, 0.3 \mathrm{~mL}$ of BTME was added, and the solid product obtained after stirring two hours was centrifuged and washed, dried at $80^{\circ} \mathrm{C}$ and calcined at $500^{\circ} \mathrm{C}$ for 3 hours. The final solid product was named $\mathrm{MSiO}_{2}$ YSNSs.

\section{S2. Preparation of $\mathrm{Pd}-\mathrm{WO}_{3} / \mathrm{MSiO}_{2}$ hollow nanospheres and $\mathrm{Pd}-\mathrm{WO}_{3} / \mathrm{SBA}-15$}

The $0.12 \mathrm{~g}$ CTAB was dissolved in distilled water $(45 \mathrm{~mL})$, and stirring for $30 \mathrm{~min}$ after heated to $95{ }^{\circ} \mathrm{C}$. Then $5 \mathrm{ml} \mathrm{K}_{2} \mathrm{PdCl}_{4}$ solution $(0.02 \mathrm{~mol} / \mathrm{L})$ was added, and when the temperature rose to $95{ }^{\circ} \mathrm{C}$, $1.7 \mathrm{ml}$ of AA solution $(0.1 \mathrm{~mol} / \mathrm{L})$ was added. After stirring for $30 \mathrm{~min}, \mathrm{MSiO}_{2}$ hollow nanospheres was added to the above solution and stirred for 3 hours, removed water and calcined at $500^{\circ} \mathrm{C}$ for three

Corresponding author. E-mail: liuyuequst@163.com (Y Liu), E-mail: liushiweiqust@126.com (SW Liu) 
hours to obtain $\mathrm{PdO} / \mathrm{MSiO}_{2}$ hollow nanospheres. Then $\mathrm{PdO} / \mathrm{MSiO}_{2} \mathrm{YSNSs}$ was added into ammonium tungstate solution, stirred at room temperature for eight hours, and then dried at $80{ }^{\circ} \mathrm{C}$ to remove water.

The solid was calcined at $500{ }^{\circ} \mathrm{C}$ for 3 hours and reduced at $200{ }^{\circ} \mathrm{C}$ for 2 hours in hydrogen atmosphere. The finally product was labeled $\mathrm{Pd}-\mathrm{WO}_{\mathrm{x}} / \mathrm{MSiO}_{2} \mathrm{YSNSs}$. Pd- $\mathrm{WO}_{3} / \mathrm{SBA}-15$ was prepared by the same method.

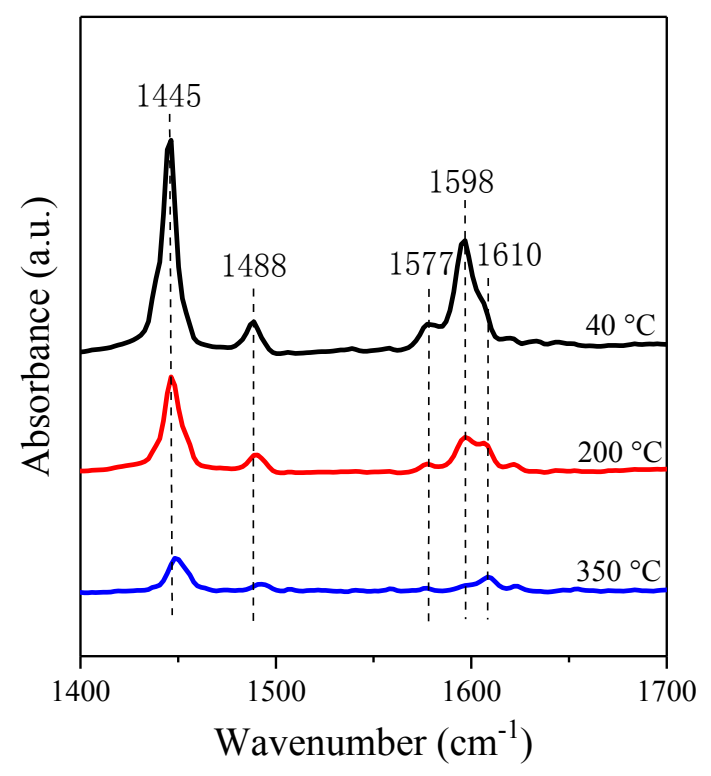

Figure S1. Py-FTIR pattern of the $\mathrm{Pd} @ 1.5 \% \mathrm{WO}_{\mathrm{x}}-\mathrm{MSiO}_{2} \mathrm{YSNSs}$.
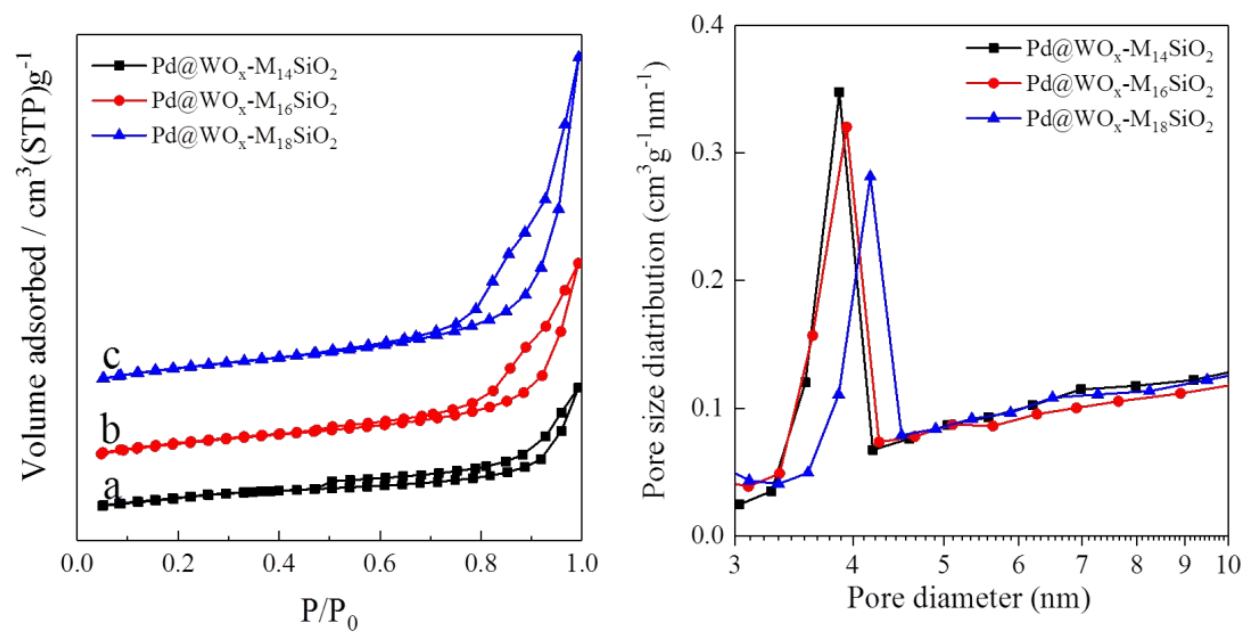

Figure S2. $\mathrm{N}_{2}$ adsorption-desorption isotherms and pore size distribution of various pore size catalysts. 

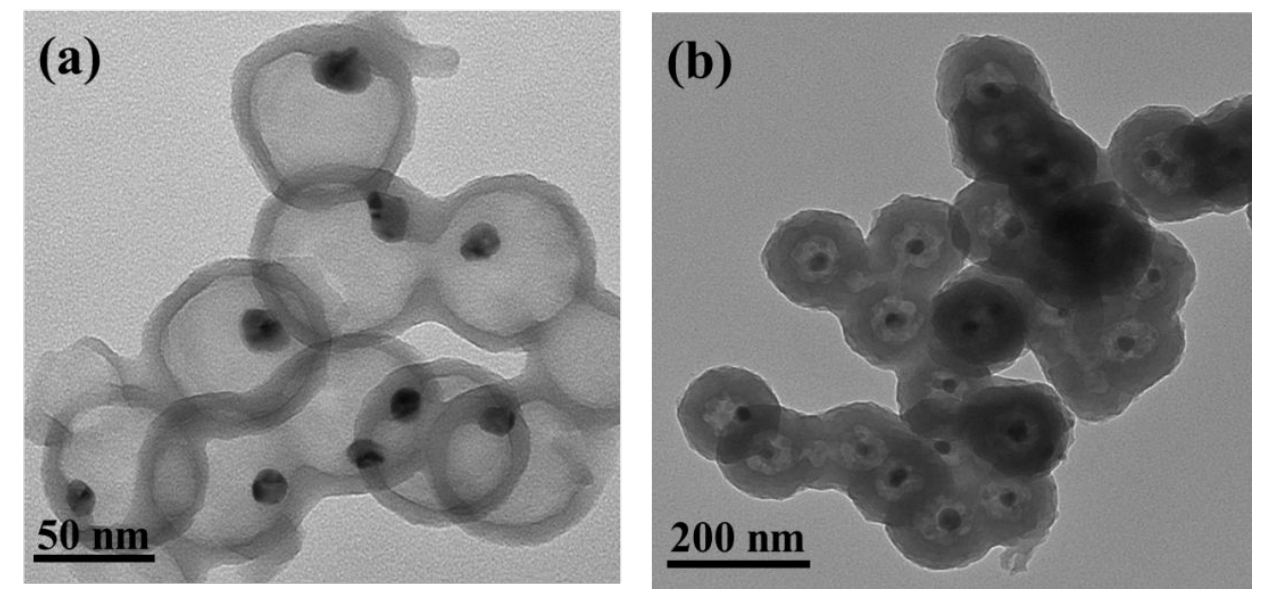

Figure S3. TEM of different shell thicknesses catalysts: (a) $\mathrm{Pd} @ \mathrm{WO}_{\mathrm{x}}-\mathrm{MSiO}_{2}-8 \mathrm{~nm} \mathrm{YSNSs}$, (b) $\mathrm{Pd} @ \mathrm{WO}_{\mathrm{x}}-\mathrm{MSiO}_{2}-38 \mathrm{~nm}$ YSNSs.
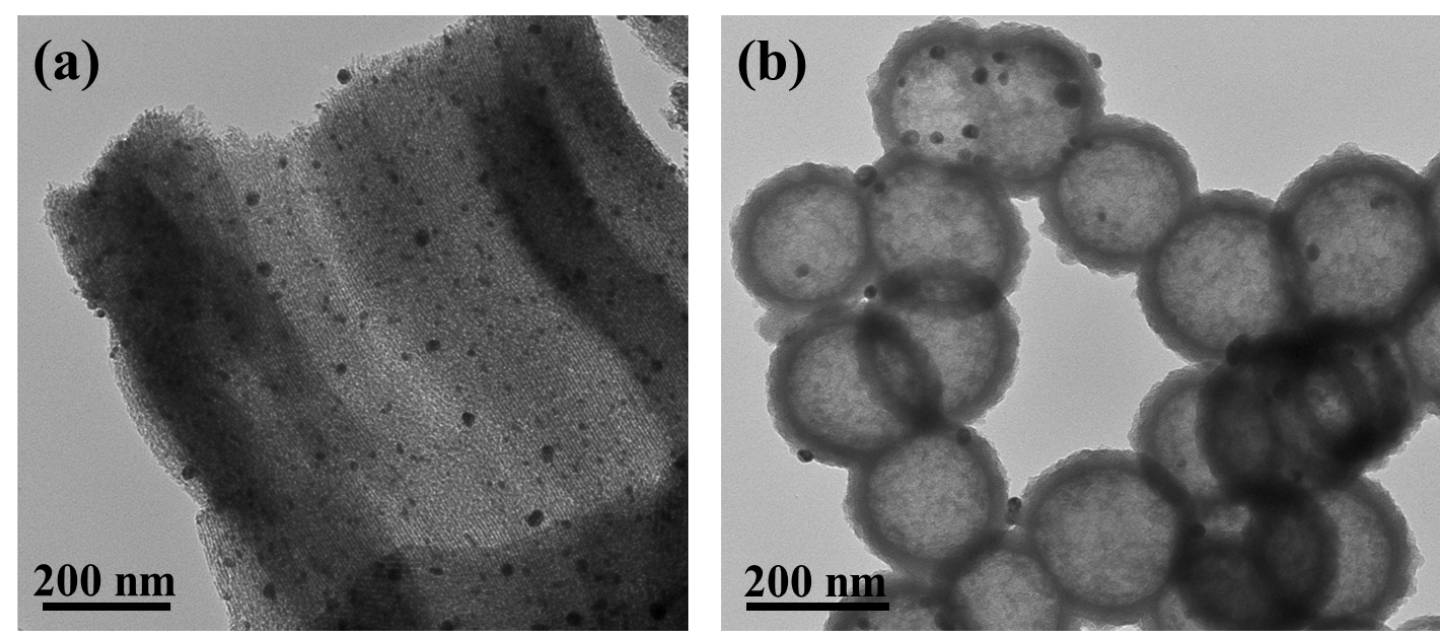

Figure S4. TEM of Pd-WO ${ }_{\mathrm{x}} / \mathrm{SBA}-15$ and $\mathrm{Pd}-\mathrm{WO}_{\mathrm{x}} / \mathrm{MSiO}_{2} \mathrm{YSNSs}$.

Table S1. Physicochemical parameters of the Pd@ $\mathrm{WO}_{\mathrm{x}}-\mathrm{MSiO}_{2}$ YSNSs 


\begin{tabular}{cccc}
\hline sample & $\mathrm{S}_{\mathrm{BET}}\left(\mathrm{m}^{2} / \mathrm{g}\right)$ & $\mathrm{V}_{\text {total }}\left(\mathrm{cm}^{3} \mathrm{~g}^{-1}\right)$ & $\mathrm{D}_{\text {peak }}(\mathrm{nm})$ \\
\hline $\mathrm{Pd} @ 0.5 \% \mathrm{WO}_{\mathrm{x}}-\mathrm{MSiO}_{2}$ & 209.9340 & 0.512278 & 3.93 \\
$\mathrm{Pd} @ 1.0 \% \mathrm{WO}_{\mathrm{x}}-\mathrm{MSiO}_{2}$ & 221.8098 & 0.507307 & 3.93 \\
$\mathrm{Pd} @ 1.5 \% \mathrm{WO}_{\mathrm{x}}-\mathrm{MSiO}_{2}$ & 224.4637 & 0.474532 & 3.92 \\
$\mathrm{Pd} @ 2.0 \% \mathrm{WO}_{\mathrm{x}}-\mathrm{MSiO}_{2}$ & 224.5928 & 0.472722 & 3.91 \\
$\mathrm{Pd} @ 2.5 \% \mathrm{WO}_{\mathrm{x}}-\mathrm{MSiO}_{2}$ & 224.8858 & 0.472985 & 3.91 \\
\hline
\end{tabular}

Table S2. The $\mathrm{NH}_{3}-\mathrm{TPD}$ parameters of $\mathrm{Pd} @ \mathrm{WO}_{\mathrm{x}}-\mathrm{MSiO}_{2} \mathrm{YSNSs}$ with different tungsten content

\begin{tabular}{|c|c|c|c|c|}
\hline sample & peak & Temperature $\left({ }^{\circ} \mathrm{C}\right)$ & Quantity $(\mathrm{mmol} / \mathrm{g})$ & Totle quantity $(\mathrm{mmol} / \mathrm{g})$ \\
\hline & peak 1 & 152.7 & 0.04591 & \\
\hline \multirow[t]{2}{*}{$\mathrm{Pd} @ 0.5 \% \mathrm{WO}_{\mathrm{x}}-\mathrm{MSiO}_{2}$} & & & & 0.11026 \\
\hline & peak 2 & 397.6 & 0.06435 & \\
\hline \multirow{3}{*}{$\mathrm{Pd} @ 1.0 \% \mathrm{WO}_{\mathrm{x}}-\mathrm{MSiO}_{2}$} & peak 1 & 147.2 & 0.04302 & \\
\hline & & & & 0.12295 \\
\hline & peak 2 & 397.3 & 0.07993 & \\
\hline \multirow{3}{*}{$\mathrm{Pd} @ 1.5 \% \mathrm{WO}_{\mathrm{x}}-\mathrm{MSiO}_{2}$} & peak 1 & 156.1 & 0.05043 & \\
\hline & & & & 0.18587 \\
\hline & peak 2 & 431.2 & 0.13544 & \\
\hline \multirow{3}{*}{$\mathrm{Pd} @ 2.0 \% \mathrm{WO}_{\mathrm{x}}-\mathrm{MSiO}_{2}$} & peak 1 & 147.2 & 0.04086 & \\
\hline & & & & 0.20059 \\
\hline & peak 2 & 415.6 & 0.15973 & \\
\hline \multirow{3}{*}{$\mathrm{Pd} @ 2.5 \% \mathrm{WO}_{\mathrm{x}}-\mathrm{MSiO}_{2}$} & peak 1 & 149.2 & 0.04003 & \\
\hline & & & & 0.22643 \\
\hline & peak 2 & 427.6 & 0.1864 & \\
\hline
\end{tabular}

Table S3. Acid parameters of the $\mathrm{Pd} @ 1.5 \% \mathrm{WO}_{\mathrm{x}}-\mathrm{MSiO}_{2}$ YSNSs. 


\begin{tabular}{ccccc}
\hline Temperature & Bronsted acid & Lewis acid & Total acid amount & Bronsted acid / \\
$\left({ }^{\circ} \mathrm{C}\right)$ & amount $(\mathrm{umol} / \mathrm{g})$ & $(\mathrm{umol} / \mathrm{g})$ & $(\mathrm{umol} / \mathrm{g})$ & Lewis acid \\
\hline 40 & 3.156833 & 145.7186 & 148.8755 & 0.021664 \\
200 & 1.384424 & 73.84 & 75.22442 & 0.018749 \\
350 & 0.621183 & 25.98245 & 26.60363 & 0.023908 \\
\hline
\end{tabular}

Table S4. Physicochemical parameters of the different pore size $\mathrm{Pd} @ \mathrm{WO}_{\mathrm{x}}-\mathrm{MSiO}_{2} \mathrm{YSNSs}$

\begin{tabular}{cccc}
\hline sample & $\mathrm{S}_{\mathrm{BET}}\left(\mathrm{m}^{2} / \mathrm{g}\right)$ & $\mathrm{V}_{\text {total }}\left(\mathrm{cm}^{3} \mathrm{~g}^{-1}\right)$ & $\mathrm{D}_{\text {peak }}(\mathrm{nm})$ \\
\hline $\mathrm{Pd} @ \mathrm{WO}_{\mathrm{x}}-\mathrm{M}_{14} \mathrm{SiO}_{2}$ & 173.6412 & 0.286834 & 3.87 \\
$\mathrm{Pd} @ \mathrm{WO}_{\mathrm{x}}-\mathrm{M}_{16} \mathrm{SiO}_{2}$ & 224.4637 & 0.474532 & 3.92 \\
$\mathrm{Pd} @ \mathrm{WO}_{\mathrm{x}}-\mathrm{M}_{18} \mathrm{SiO}_{2}$ & 246.2824 & 0.672669 & 4.17 \\
\hline
\end{tabular}

